\title{
Effect of structured visual environments on apparent eye level
}

\author{
ARNOLD E. STOPER \\ California State University, Hayward, California \\ and \\ MALCOLM M. COHEN \\ NASA-Ames Research Center, Moffett Field, California
}

\begin{abstract}
Each of 12 subjects set a binocularly viewed target to apparent eye level; the target was projected on the rear wall of an open box, the floor of which was horizontal or pitched up and down at angles of $7.5^{\circ}$ and $15^{\circ}$. Settings of the target were systematically biased by $60 \%$ of the pitch angle when the interior of the box was illuminated, but by only $5 \%$ when the interior of the box was darkened. Within-subjects variability of the settings was less under illuminated viewing conditions than in the dark, but was independent of box pitch angle. In a second experiment, 11 subjects were tested with an illuminated pitched box, yielding piases of $53 \%$ and $49 \%$ for binocular and monocular viewing conditions, respectively. The results are discussed in terms of individual and interactive effects of optical, gravitational, and extraretinal eye-position information in determining judgments of eye level.
\end{abstract}

It has long been known that a structured visual environment (SVE) has a strong influence on judgments of the gravitational vertical and horizontal. In most studies of this effect, the roll dimension has been investigated-as, for example, in the now classical "rod and frame" paradigm (Witkin \& Asch, 1948). The effect has been variously labeled, as a "visual bias of response" (Kaufman, 1974), a "righting" effect (Rock, 1983), or the "rod and frame effect"' (Howard, 1982)-or, again, when the optical information completely dominates the gravitational, it has been called "visual capture"' (Hay, Pick, \& Ikeda, 1965; Tastevin, 1937). Bischof (1974) uses the term "optic-vestibular weight ratio" to refer to the effect's magnitude. Here, we will refer to this as an "optical bias of response," or simply, "optical bias."

In contrast to the voluminous literature concerning the roll dimension, experimental investigations of optical bias in the pitch dimension-for example, of the optical bias of judgments of eye level-have been very few (see Howard, 1982, for reviews). Recently, however, Matin and his associates (Matin \& Fox, 1986, 1989; Matin, Fox, \& Doktorsky, 1987) have pointed out that the effect of

This research was supported by funds under NASA Research Project 199-22-22-16, Grant NCC2-318. We thank Gail Adams and Kristen Lindseth for data collection, editorial comments, and support, and Tom Palmer and Geoff Apple for technical assistance. We would also like to thank Leonard Matin, who graciously allowed us to visit his laboratory in 1985, and who subsequently communicated to us his unpublished research results. We had originally proposed to pitch the structured visual environment by means of mirrors; however, on viewing Dr. Matin's "pitchroom" we were inspired to use the "pitchbox" method described here. Correspondence may be addressed to Arnold E. Stoper, Department of Psychology, California State University, Hayward, CA 94542. an SVE on eye level is of high theoretical interest, and that it is closely related to the more general problem of taking eye position into account in the determination of visual direction. Matin et al. (1982) demonstrated that curarized subjects make large errors in the localization of a luminous target, with respect to both eye level and the median plane, but only in the absence of an SVE. Evidently, the extent to which extraretinal factors are used in the determination of visual direction changes with the presence or absence of an SVE. In the present paper, we explore the nature of the interaction of visual and gravitational stimuli in the determination of eye level.

An observer's eye level may be defined as a plane that passes through his or her eyes; this plane is, in some sense, "level." There are, however, at least three distinct meanings for the term level, and hence there are at least three distinct types of eye level: (1) eye level that is parallel to the transverse plane of the head, which we will call head-referenced eye level (HREL); (2) eye level that is normal to the direction of gravity, which we will call gravity-referenced eye level (GREL); and (3) eye level that is parallel to the ground plane or the longitudinal axis of the SVE, which we will call structure-referenced eye level (SREL).

The physiological systems underlying judgments of eye level will depend on different sources of information, depending on the type of eye level involved. Both HREL and GREL judgments require information about the position of the target image on the retina and information about the position of the eye in the head. We call the physiological system responsible for the determination of the position of the eye in the head the eye/head system (after Gregory, 1958). Presumably this system works by means of extraretinal eye-position information (Matin, 1982). 
GREL judgments, in addition to information about the position of the target on the retina and the position of the eye in the head, require information about the position of the head with respect to gravity. We call the physiological system responsible for this determination the head/gravity system. It presumably works by means of vestibular (primarily otolithic) and postural information (Cohen, 1973; Cohen \& Larson, 1974; Graybiel, 1956). The determination of GREL thus requires the use of both the eye/head and the head/gravity systems; we call this combined system the eye/head/gravity system.

SREL judgments require neither eye/head nor head/ gravity information. No extraretinal, vestibular, or other proprioceptive information is necessary to determine this eye level; it can be determined completely by optical information alone. The optical information involved might be in the form of depth cues that allow the observer to compare eye-to-surface distance with target-to-surface distance, thus determining points that are at the same height from the ground surface as in the observer's eye. Alternatively, the optical information might be in a form that allows a direct determination of SREL without recourse to judgments of distance. For example, the visible horizon (the line between the ground plane and the sky) may directly specify SREL (Gibson, 1950, 1966; Purdy, 1958; Sedgwick, 1980). We will call the physiological system responsible for determining SREL the optical pattern system. In principal, the optical pattern system can be completely independent of the eye/head/gravity system.

When a subject stands on a level ground plane in a normally illuminated terrestrial environment, with head erect, all three eye levels coincide, and determination of any one thus provides determination of the other two. This fact allows for a redundancy that could be advantageous to the organism. The experiment by Matin et al. (1982), for example, shows that with the eye/head/gravity system incapacitated by curare, a subject can nevertheless accurately determine GREL in an illuminated environment. Presumably subjects can do this by making use of the optical pattern system, which evidently can serve as a "backup" for the eye/head/gravity system.

The optical bias effect shows that the involvement of the optical pattern system can, under some conditions, lead to errors in judgment of GREL. The assumption underlying the use of the term optical bias is that the head/gravity system can provide an "unbiased" signal, perhaps with its own inaccuracies (as in Matin's experiment). The judgment of GREL can become "biased" in the direction of SREL through the addition of a conflicting signal from the optical pattern system.

We express the magnitude of the optical bias effect as a bias ratio, which is defined as the ratio of the actual measured bias to the total possible bias. In general, the total possible bias would be the angular difference between an unbiased apparent GREL and a true SREL. An unbiased apparent GREL could be determined by measuring it in the dark, or, in some circumstances, by simply assuming that it is equal to true GREL. A bias ratio of
1.00 would indicate that the optical pattern system totally determines judged GREL, whereas a bias ratio of zero would indicate that the optical pattern system has no influence on judged GREL.

Using this measure, we can compare the results of various studies in which optical bias of judgments in the pitch dimension has been found. The result reported by Matin et al. (1982), cited above, suggests that curarized subjects have virtually complete capture and hence a bias ratio approximating 1.00. Asch and Witkin (1948a), using a natural scene viewed through a sloping mirror, obtained data that suggest a bias ratio of .88. Rock, Goldberg, and Mack (1966), who had subjects judge eye level through a prism with a deviation of $11^{\circ}$, found similar results: the bias ratio for their data is approximately .70. MacDougall (1903) and Perrone (1977), both using a more restricted SVE, found a much smaller effect (a bias ratio of .10).

More recently, Matin and Fox $(1986,1989)$ used a pitchroom, a large rectangular structure that surrounded the subject. The pitchroom was mounted on rockers to allow pitching of $40^{\circ}$ up or down from the horizontal. With this arrangement, they obtained data with a bias ratio of .56. Kleinhans (1970) used a smaller rectangular structure, which we will call a pitchbox. This is simply a box open on the viewing end, capable of being pitched up and down. In Kleinhans's experiment, the range of pitch was $\pm 25^{\circ}$, and we have computed a bias ratio of .44 from his data.

Harris (1974) suggested that optical bias in the pitch dimension can be explained as a "straight-ahead shift." This is defined as a change in the egocentric spatial direction, which the subject treats as being straight ahead or, in this case, at eye level. Our discussion of three possible eye levels and the confusion among them (especially confusion between GREL and SREL in the case of optical bias) is consistent with a straight-ahead shift hypothesis. However, Harris further defined the straight-ahead shift as being a cognitive, rather than a perceptual, effect. (A perceptual effect, according to Harris, would result, for example, in a pointing error as well as a shift in apparent egocentric direction.)

Although Harris did not say so explicitly, confusion between discordant determinations of eye level might be expected to result in increased variability of judgments. One might expect, then, that the larger the discordance between GREL and SREL (i.e., the larger the pitch angle of the box), the larger the variability of judged GREL. Our Experiment 1 was intended, in part, to check this expectation.

\section{EXPERIMENT 1}

\section{Method}

A modification of the pitchbox method (Kleinhans, 1970) was used.

\section{Apparatus}

Pitchbox. Each subject was seated in front of a large chamber (pitchbox), which was constructed of Styrofoam and rested on a plywood base. The inside of the pitchbox was $72 \mathrm{~cm}$ high, $72 \mathrm{~cm}$ 

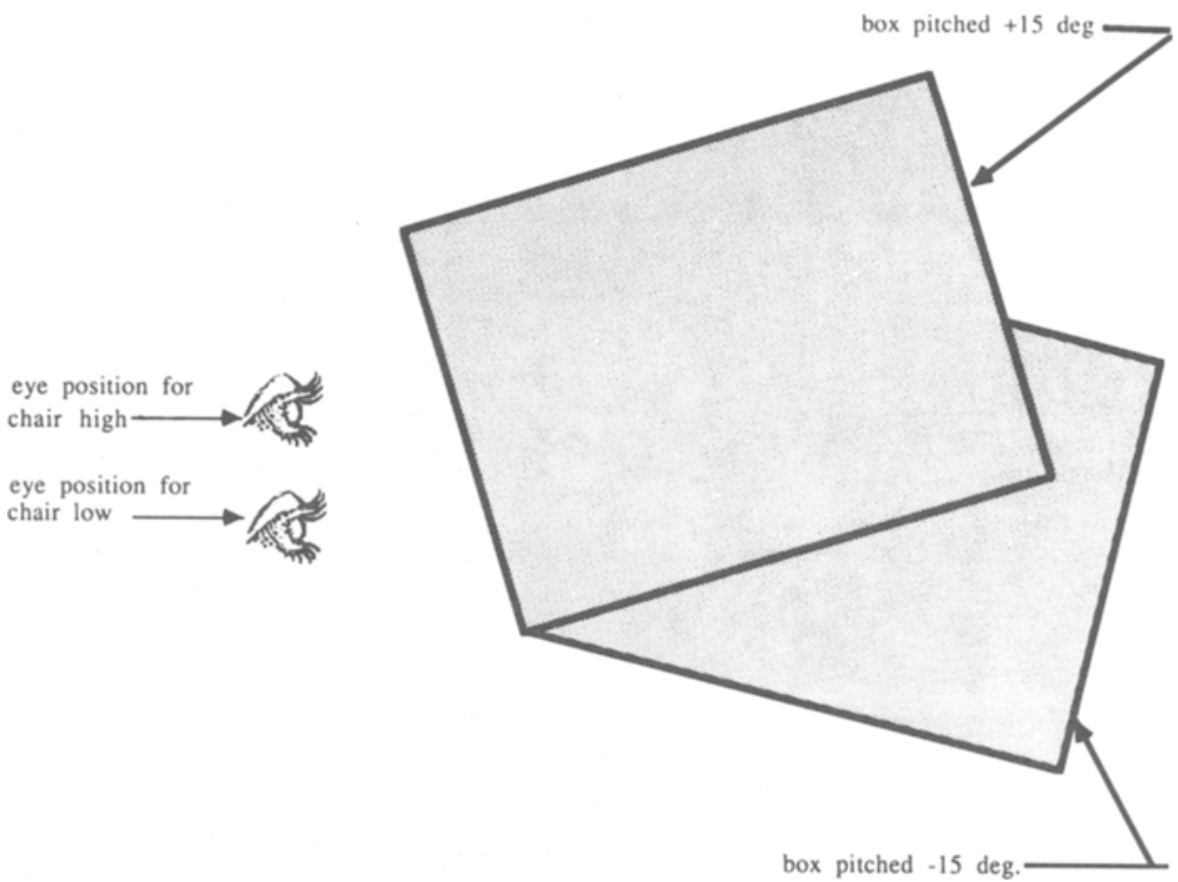

Figure 1. Pitchbox viewing positions.

wide, and $115 \mathrm{~cm}$ deep. The subject was seated with head laterally centered in front of the box so that the eyes were $132 \mathrm{~cm}$ from the back of the box when the box was level (see Figure 1). All of the internal surfaces of the pitchbox were lined with matte black posterboard to minimize any internal reflections of the target that might have furnished visual cues in the dark condition. The posterboard also served to minimize any surface markings in the pitchbox that might have been used as cues in the light condition.

Illumination. For lighted viewing conditions, illumination was provided by a diffused $15-\mathrm{W}$ incandescent lamp; the luminance of the back wall of the pitchbox was $1 \mathrm{~cd} / \mathrm{m}^{2}$. The light was fixed to the movable pitchbox rather than to the external environment to prevent the use of changes in shading as cues for pitchbox orientation. For the dark viewing condition, no extraneous sources of illumination were visible to the subject.

Target. The target, a pinpoint image ( $<2 \mathrm{~mm}$ in diameter) provided by a $0.5 \mathrm{~mW} 632 \mathrm{~nm}$ laser (Spectra Physics Model 155), was projected in the subject's midsagittal plane onto the back wall of the pitchbox. The laser beam was attenuated to $10 \%$ of its original intensity by a neutral density filter, reducing the possibility of having scattered light illuminate surfaces in the pitchbox.

The laser, mounted on a photographic tripod, was free to rotate about its pitch axis. This pitch motion was controlled by a wormgear apparatus connected to a handle by means of a flexible shaft. The subject could move the target up and down by rotating this handle. This arrangement was designed to minimize kinesthetic cues regarding the orientation of the laser.

The orientation of the laser was monitored by means of a pointer fixed to the laser but projecting its "point" against the wall behind, and invisible to, the subject. The position of this point measured with respect to the floor of the room was used as a measure of target position. The point position was related to the target position by means of an independent calibration procedure at each pitchbox orientation.

Chair. Throughout the study, the subject was seated in a dental chair, as described in detail in Stoper and Cohen, 1986. The height of the chair could be adjusted by the experimenter with footpedal controls; it was varied to minimize the possibility that the subject would artificially increase his or her precision through the use of background details as cues or anchors.

The dental chair was provided with an adjustable headrest, against which the subject was instructed to keep his or her head. Although no additional restraints were used, the headrest effectively restricted pitch movements of the head. Head pitch was monitored with the aid of goggles, LEDs, and a video system. Motions of each subject's head about the roll axis were neither restrained nor monitored; it was assumed that these motions would not systematically influence judgments of eye level. There was no need to fix the vertical position of the subject's head with respect to the chair, since the important variable was the height of the eyes relative to the target, and this was measured by the video system. The chair was positioned at either of two fixed heights, separated by $7.6 \mathrm{~cm}$ (see Figure 1). The high chair position placed the subject's eyes $40.6 \mathrm{~cm}$ above the axis of rotation of the pitchbox.

Goggles. All viewing was binocular. Each subject wore clear transparent protective goggles with an oval faceplate that measured $10 \times 6 \mathrm{~cm}$; the goggles restricted the subject's field of vision to the interior of the box. Two LEDs were mounted approximately $1 \mathrm{~cm}$ apart on the outside of the goggles, along a horizontal line from front to back. The LEDs, not visible to the subject, were used by the experimenter to monitor both the height of the eyes and to assure the continuous upright orientation of the subject's head.

Video system. A video image of the goggle-mounted LEDs was used to determine the height of each subject's eyes and the orientation of the subject's head, both in the dark and in the light.

\section{Procedure}

Subjects. Twelve subjects participated in this study, 6 males and 6 females. Eight subjects were naive to the purpose of the experiment; the remaining 4 were the authors of this paper and their assistants.

Instructions. Each subject was instructed to find the point in the pitchbox that was at his or her eye level, and adjust the height of 
the target until it was at this point. Eye level was defined as the point in view when the line of sight was parallel to floor of the room in which the subject was sitting. The subject was further instructed to keep the head steady against the headrest, with eyes closed at all times except when adjusting the target height.

After each setting was made, the experimenter reminded the subject to close his or her eyes. The experimenter then moved the target to a new starting position, near either the top or the bottom of its range, repositioned the pitchbox to one of five orientations, and adjusted the chair height to one of the two fixed positions. In the rare event that a change of the subject's head position was detected on the video monitor, the experiment was momentarily stopped and the subject was instructed to reposition the head.

Data collection. The experiment consisted of four factors: (1) orientation, that is, boxpitch $\left(15^{\circ}\right.$ up, $7.5^{\circ}$ up, $0^{\circ}$ level, $7.5^{\circ}$ down, and $15^{\circ}$ down), (2) illumination (light and dark), (3) chair position (high and low), and (4) laser movement (up and down; i.e., starting position of the laser either low or high), yielding a total of $\mathbf{4 0}$ trials per subject. The trials were presented in blocks of 10 , consisting of the five orientations presented twice in counterbalanced order. A block of 10 trials was presented in the dark, followed by another block in the light, followed by at least a 5-min break, then dark and light again. This sequence was used to minimize the influence of a light condition on a following dark condition. Five trials in a block were presented at one chair position, followed by five at the other. This order was used to minimize any up and down movements of the subject. The laser conditions were alternated for each trial.

\section{Results}

A four-way ANOVA was applied to the data, and a strong main effect of orientation $[F(4,44)=47.6, p<$ $.0001]$, as well as a strong interaction between orientation and illumination $[F(4,44)=57.5, p<.0001]$, was obtained. These two effects can be seen clearly in Figure 2, where mean bias of judged GREL is plotted as a function of orientation of the pitchbox. It is clear that a strong effect of orientation on GREL exists in the light condition, but not in the dark. The slope of this function represents the optical bias ratio. It is calculated to be .60 in the light,

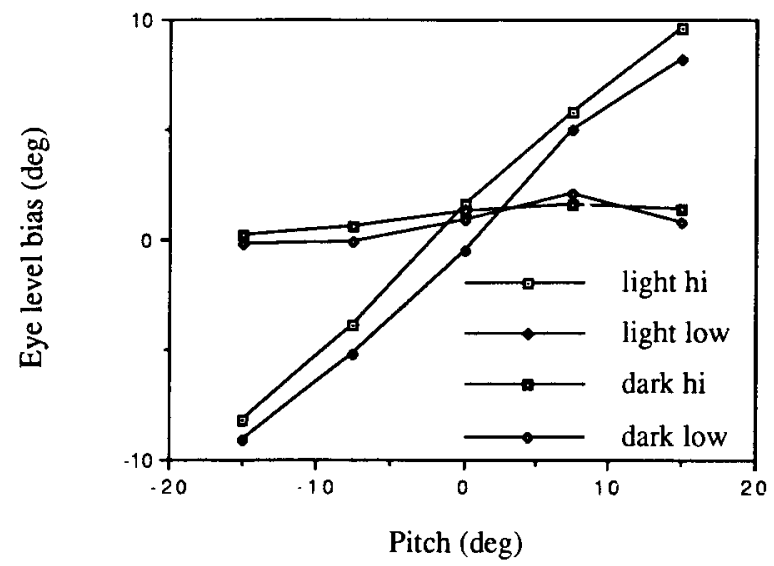

Figure 2. Mean bias in judgment of GREL for 12 subjects, as a function of pitchbox orientation and illumination as well as eye position.

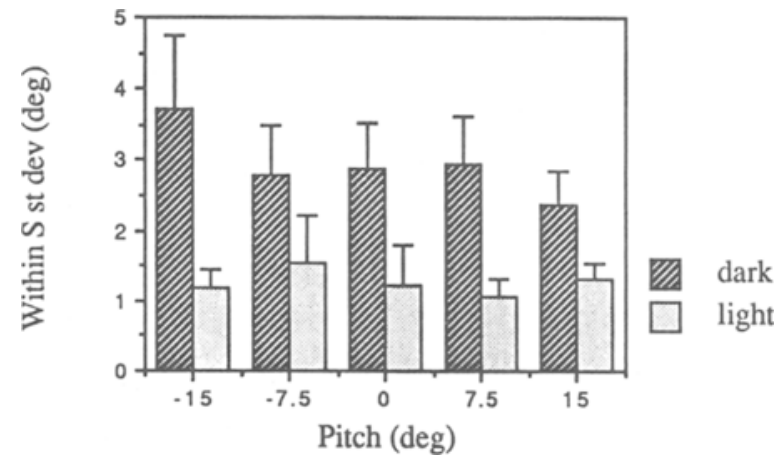

Figure 3. Standard deviations (within-subjects) of GREL judgments of 12 subjects for each of 5 pitchbox orientations, in the light and in the dark. Error bars represent standard error of the mean.

and .05 in the dark. The orientation effect is essentially linear, indicating that the bias ratio was constant over the five orientations.

\section{Chair Height}

Separate three-way ANOVAs were run on data from the light and dark conditions. Orientation was significant both in the light $[F(4,44)=60.6, p<.0001]$ and in the dark $[F(4,44)=3.4, p<.02]$. Chair position had a significant effect $[F(1,11)=11.9, p<.01]$ in the light, but not in the dark. This effect can also be seen in Figure 2. There is a mean difference in GREL bias of $1.3^{\circ}(2.9 \mathrm{~cm})$ between the high and the low chair positions (or about $40 \%$ of the $7.6-\mathrm{cm}$ vertical shift in eye position).

\section{Variability}

Calculation of standard deviations indicated some interesting patterns. The overall within-subjects standard deviations, pooling over all orientations, was $5.24^{\circ}$ in the light, and $3.1^{\circ}$ in the dark. Although this appears to be in sharp contrast to results obtained with completely level surroundings (Stoper \& Cohen, 1986), which indicated less variability in the light $\left(S D=1.03^{\circ}\right)$ than in the dark $\left(S D=1.72^{\circ}\right)$, Figure 3 shows that when within-subjects standard deviations are calculated separately for each of the five orientations, in the light and the dark (thus removing variability due to the orientation effect), average variability in the light $\left(S D=1.26^{\circ}\right)$ is in fact much less than in the dark $\left(S D=2.94^{\circ}\right)$. An ANOVA applied to the standard deviation data showed this effect of illumination to be highly significant $[F(1,44)=58.3, p<.0001]$ but showed no significant effect of orientation, either in the light or in the dark.

Figure 4 shows the 40 judgments plotted in temporal sequence. It should be recalled that, during both dark and light blocks of trials, the pitchbox was moved sequentially through the five orientations from $-15^{\circ}$ to $+15^{\circ}$, then back from $+15^{\circ}$ to $-15^{\circ}$. This down and then up pattern can be seen clearly in the light trials of Figure 4 . For both blocks of dark trials (Trials 1 through 10 and 21 through 


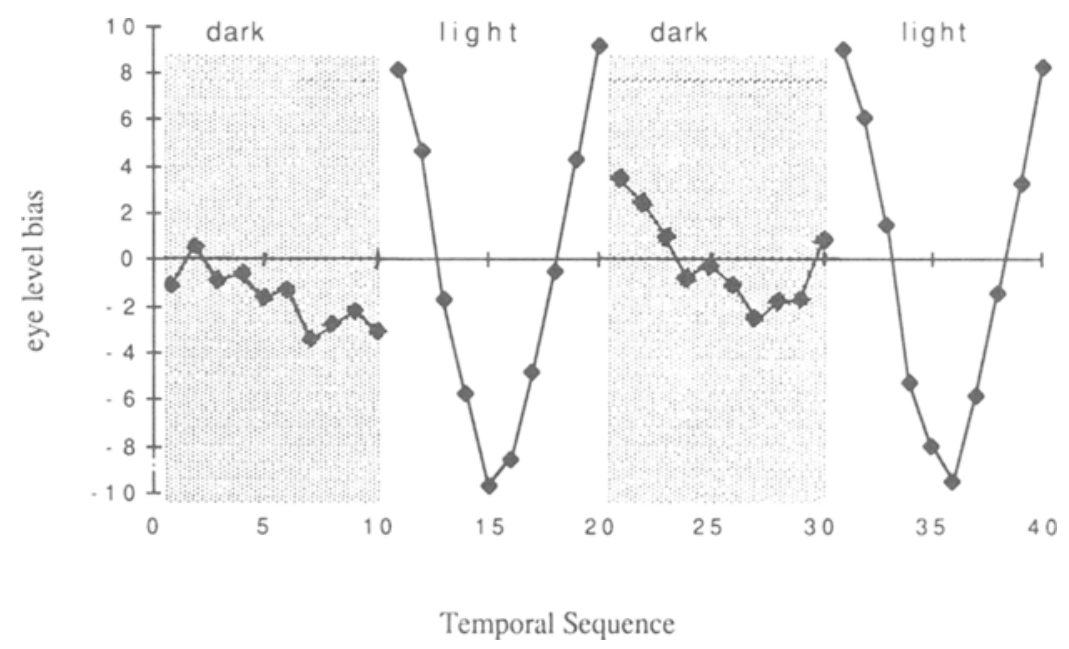

Figure 4. Mean bias in judgment of GREL for 12 subjects, plotted in temporal sequence.

30), there is a downward drift over time; comparison of the first trial of a particular orientation with the second trial at that orientation showed the second trial to be an average of $2.7^{\circ}$ lower (significant by sign test; $p<.001$ ).

\section{Discussion}

Contrary to the expectation of increased variability with increase in deviation of the pitchbox from level, we found no effect of pitchbox orientation on within-subjects variability. Variability when the pitchbox was level, at $0^{\circ}$, was just as great as when it was at maximum deviation from level, at $\pm 15^{\circ}$. Subjects did not seem to fluctuate from one "meaning" of eye level to another, nor even to have any large fluctuation in weighting (bias ratio). The eyelevel judgment seems to be determined by compromise between the eye/head/gravity system and the optical pattern system, and there is a remarkably stable weight given to each system, independently of boxpitch orientation. This seems to be the result of an automatic, unconscious, perceptual mechanism, rather than the cognitive mechanism hypothesized by Harris.

\section{Pitch}

The effect of pitchbox orientation in the light is comparable in magnitude to that found by Kleinhans (1970) and by Matin and Fox (1988). The effect of orientation was very much smaller in the dark than in the light; it was nevertheless statistically significant. Somehow, even in what we had assumed to be complete darkness, information about pitchbox orientation must have been available to the subjects. One possible source of this information may have come from the laser target itself; as the subjects moved the laser past an inside edge of the pitchbox, the apparent path of movement was deflected. This deflection could have revealed to the subjects the location of the edge.
The linearity of the orientation effect (i.e., the constancy of bias ratio over orientation) is consistent with the findings of Kleinhans (1970) and of Matin and Fox (1988).

\section{Chair Height}

The effect of chair height will be called the linear displacement effect. The rotation of the pitchbox around its axis, at the lower front edge, which was $115 \mathrm{~cm}$ from its back, can be considered to produce two separate effects. Not only is orientation changed, but the back of the pitchbox is displaced vertically with respect to the eye. With a $15^{\circ}$ upward pitch, for example, the bottom rear edge at the back of the box is displaced $29.8 \mathrm{~cm}$ upward. Assuming that the linear displacement effect of roughly $40 \%$ found here for the 7.6- $\mathrm{cm}$ displacement of the chair holds also for the $29.8-\mathrm{cm}$ displacement, the $29.8-\mathrm{cm}$ displacement would produce a shift in judged GREL of $11.9 \mathrm{~cm}$, which is $5.2^{\circ}$ of visual angle at the viewing distance of $132 \mathrm{~cm}$. Thus, approximately $65 \%$ of the $8^{\circ}$ shift in GREL found for the $15^{\circ}$ box pitch might be accounted for by the linear displacement of the back of the box.

It is possible that the linear displacement effect is an instance of the "Dietzel-Roelofs" effect, in which the apparent straight ahead is displaced toward the center of an asymmetrical visual display (see Howard, 1982).

\section{Variability}

The standard deviations obtained in the light are comparable to those obtained by Stoper and Cohen (1986) for level surroundings. Those obtained here in the dark, however, are much higher than in the previous study. This discrepancy may be due to different trial-presentation sequences in the two studies. In the 1986 study, light and dark trials were alternated. Here, in order to minimize the effect of light trials on following dark trials, a block of 10 dark trials was run followed by a block of 10 light 
trials. Each block of 10 trials occupied approximately $10 \mathrm{~min}$. As our time sequence data show, there was considerable drift during those $10 \mathrm{~min}$, and this drift would contribute to increased variability.

The fact that eye level tended to drift down during the dark trials is something of a mystery, and we have no satisfactory explanation for this finding. We can rule out any artifact due to actual chair movement; this would have been easily detected by the head-motion monitoring method used.

The long block of dark trials might be expected to produce a strong autokinetic effect, which could contribute to drift of eye level. Such an effect was seldom reported, however, and in exploratory studies, we found it to be minimal under these conditions. Having subjects control the motion of the point may have minimized autokinesis; Lackner and Zabkar (1977) found that allowing subjects to touch the light source decreased the magnitude of the autokinetic effect.

Eleven of the 12 subjects yielded a bias ratio between .5 and .7 . The remaining subject had a bias ratio of .1 . This pattern is similar to that in the data of Asch and Witkin (1948a), from which we compute that 23 of their 24 subjects had bias ratios between .7 and 1.0 , while 1 had a bias ratio of .15. Our one deviant subject was retested, and consistently showed an absence of visual capture; he was almost totally "field independent." This pattern of results suggests that, in addition to a possible continuum of field dependence-independence, some subjects use a distinctly different strategy for judging GREL.

\section{EXPERIMENT 2}

Various factors have been found to influence the extent of the optical bias effect in rod and frame experiments. Ebenholtz (1977) found that increasing the retinal size of the frame increases the effect; Asch and Witkin (1948) found that tilting the observer's body also increases it. Large individual differences have been found, with sub-

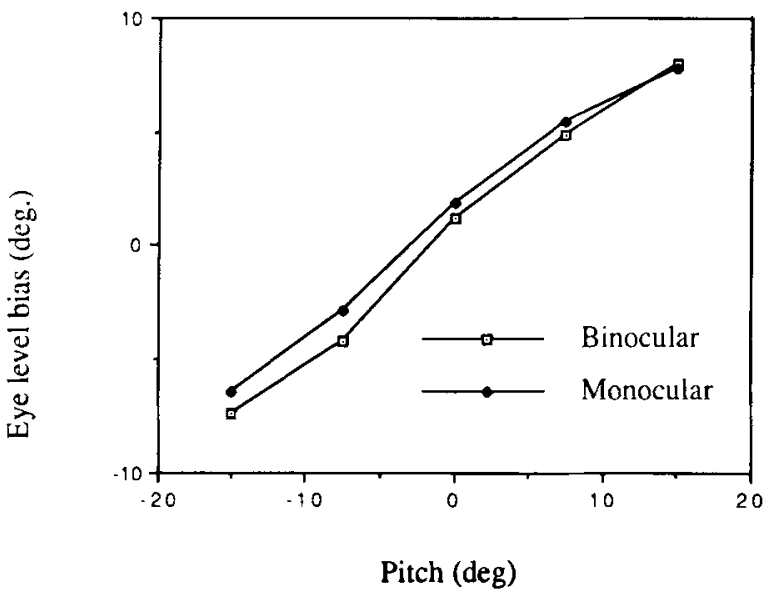

Figure 5. Mean bias in judgment of GREL for 12 subjects, as a function of pitchbox orientation and monocular versus binocular viewing.

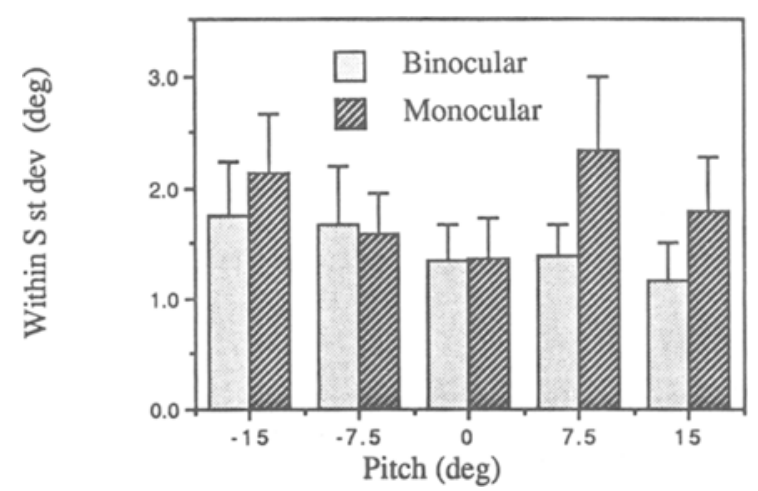

Figure 6. Standard deviations (within-subjects) of GREL judgments of 12 subjects for each of 5 pitchbox orientations, with monocular and binocular viewing. Error bars represent standard error of the mean.

jects who show a large bias being termed "field dependent" (Witkin et al., 1973). One additional factor that might be expected to have an effect on optical bias in the pitch dimension, but not in roll, is the presence of binocular cues; these could provide information about the pitch of the surface, and perhaps increase precision of judgment. In a multisensory task, the higher the precision of the sense modality, the greater the weight it may be given in determining the final percept (Bischof, 1974; Brunswik, 1956).

\section{Method}

The method was identical to that of Experiment 1, except that all trials were run in the light; half were run with monocular viewing (left eye occluded), and half with binocular viewing. The order of presentation was that of Experiment 1, with monocular trials substituted for dark trials and binocular trials substituted for light trials. Thus, 10 monocular trials were followed by 10 binocular, then 10 more monocular and 10 more binocular.

\section{Results}

A four-way ANOVA was applied to the data. A strong main effect of pitchbox orientation $[F(4,40)=86.3, p<$ $.0001]$, as well as a weak interaction between pitchbox orientation and monocular versus binocular viewing $[F(4,40)=2.69, p<.05]$, was obtained. The mean bias of judged GREL is plotted as a function of orientation of the pitchbox in Figure 5. The slope of this function represents the optical bias ratio. It is calculated to be .49 for monocular viewing, and .53 for binocular. As in Experiment 1, the orientation effect is essentially linear, indicating that the bias ratio was constant over the five orientations.

Main effects of view $[F(1,10)=6.32, p<.05]$ and chair height $[F(1,10)=19.45, p<.002]$ were also shown. The mean judged GREL for binocular viewing was $.67^{\circ}$ high; for monocular it was $.21^{\circ}$ low. The difference in judged GREL between the two chair positions was $1.25^{\circ}$, almost identical to that obtained in Experiment 1.

Figure 6 shows within-subjects standard deviations, calculated separately for each of the five orientations, for monocular and binocular viewing. Binocular viewing is slightly less variable $(S D=1.31)$ than monocular $(S D=$ 
1.82), but an ANOVA failed to show a significant difference $[F(1,44)=3.77, p<.08]$.

\section{Discussion}

Since precision of binocular judgments was not significantly higher than that of monocular judgments, one might conclude that monocular cues, such as linear perspective, can provide all of the information needed to determine surface orientation. Because of the similarity between monocular and binocular judgments, it is also not surprising that the bias ratio for monocular judgments of GREL was virtually the same as that for binocular judgments.

\section{REFERENCES}

Asch, S. E., \& WITKIN, H. A. (1948a). Studies in space orientation: I. Perception of the upright with displaced visual fields. Journal of Experimental Psychology, 38, 325-337.

Asch, S. E., \& WITkIN, H. A. (1948b). Studies in space orientation: II. Perception of the upright with displaced visual fields and with body tilted. Journal of Experimental Psychology, 38, 455-477.

Bischof, N. (1974). Optic vestibular orientation to the vertical. In H. H. Kornhuber (Ed.), Handbook of sensory physiology: (Vol. VI/2). Vestibular System (pp. 155-190). New York: Springer-Verlag.

Brunswi, E. (1956). Perception and the representative design of psychological experiments. Berkeley, CA: University of California Press.

CoHEN, M. M. (1973). Elevator illusion: Influences of otolith organ activity and neck proprioception. Perception \& Psychophysics, 14, 401-406.

COHEN, M. M., \& LARSon, C. A. (1974). Human spatial orientation in the pitch dimension. Perception \& Psychophysics, 16, 508-512.

EbENHOLTZ, S. M. (1977). Determinants of the rod and frame effect: The role of retinal size. Perception \& Psychophysics, 22, 531-538.

GiBson, J. J. (1950). The perception of the visual world. Boston: Houghton Mifflin.

Giason, J. J. (1966). The senses considered as perceptual systems. Boston: Houghton Mifflin.

Graybiel, A. (1956). The importance of the otolithic organs in man based upon a specific test for utricular function. Annals of Otology, 65, 470-487.

GREGORY, R. L. (1958). Eye movements and the stability of the visual world. Nature, 182, 1214-1216.

HARRIS, C. S. (1974). Beware of the straight-ahead shift-a nonperceptual change in experiments on adaptation to displaced vision. Perception, 3, 461-476.

HAY, J. C., PiCK, H. L., JR., \& IkedA, K. (1965). Visual capture produced by prism spectacles. Psychonomic Science, 2, 215-216.

HowARD, I. P. (1982). Human visual orientation. New York: Wiley.

Kaufman, L. (1974). Sight and mind. New York: Oxford University Press.
Kleinhans, J. L. (1970). Perception of spatial orientation in sloped. slanted and tilted visual fields. Unpublished doctoral dissertation, Rutgers University, New Brunswick, NJ.

Lackner, J. R. Zabkar, J. J. (1977). Proprioceptive information about target location suppresses autokinesis. Vision Research, 17. 1225-1229.

MacDougall, R. (1903). The subjective horizon. Psychological Review' Monograph Supplement, 4(1, Whole No. 17), 145-166.

Matin, L. (1982). Visual localization and eye movements. In A. H. Wertheim, W. A. Wagenaar, \& H. W. Leibowitz (Eds.), Tutorials in motion perception (pp. 101-156). New York: Plenum.

Matin, L., Fox, C. R. (1986). Perceived eye level: Elevation jointly determined by visual field pitch, EEPI and gravity. Investigative Ophthalmology \& Visual Science, 27 (suppl.), p. 33.

Matin, L., \& Fox, C. R. (1989). Visually perceived eye level and perceived elevation of objects: Linearly additive influences from visual field pitch and gravity. Vision Research, 29, 315-324

Matin, L., Fox, C. R., \& Doktorsky, Y. (1987). How high is up? II. Investigative Ophthalmology \& Visual Science, 26 (Apr. suppl.), p. 300 .

Matin, L., Picoult, E., Stevens, J. K., Edwards, M. W., Jr., Young, D., \& MAcArThuR, R. (1982). Oculoparalytic illusion: Visual-field dependent spatial localizations by humans partially paralyzed with curare. Scientce, 216, 198-201.

Perrone, J. A. (1977). The visual horizon and eye level judgements: A psychophysical approach to spatial orientation perception. Unpublished master's thesis, University of Canterbury.

PurdY, W. C. (1958). The hypothesis of psychophysical correspondence in space perception. (Doctoral dissertation, Cornell University, 1958) Dissenation Abstracts International, 19, 1455. (University Microfilms, No. 58-5594).

Rock, I. (1983). The logic of perception. Cambridge, MA: MIT Press.

Rock, I., GoldberG, J., \& MACK, A. (1966). Immediate correction and adaptation based on viewing a prismatically displaced scene. Perception \& Psychophysics, 1, 351-354.

SEDGWICK, H. A. (1980). The geometry of spatial layout in pictorial representation. In M. Hagen (Ed.), The perception of pictures (pp. 3338). New York: Academic Press.

Stoper, A. E., Cohen, M. M. (1986). Judgments of eye level in light and darkness. Perception \& Psychophysics, 40, 311-316.

TASTEVIN, J. (1937). En partant de l'experience d'Aristotle. LEncephale, 1, 57-84, 140-158.

WITKIN, H. A., A AsCh, S. E. (1948). Studies in space orientation: IV. Further experiments on perception of the upright with displaced visual fields. Joumal of Experimental Psychology, 38, 762-782.

Witkin, H. A., Oltman, P. K., Cox, P. W., Ehrichman, E., HAMm, R. R., \& RINGLER, R. W. (1973). Field dependence and psychological differentiation: A bibliography through 1972 with index. Princeton, NJ: Educational Testing Service Research Bulletin, 73-62.

(Manuscript received November 14, 1988; revision accepted for publication May 12, 1989.) 